\title{
Neuroendocrine carcinoma of the esophagus: Report of a case and review of the literature
}

\author{
Keisuke Kubota $^{1 *}$, Akihiro Okada ${ }^{1}$, Junko Kuroda ${ }^{1}$, Masashi Yoshida ${ }^{1}$, Nobuto Origuchi ${ }^{1}$, \\ Keiichiro Ohta ${ }^{1}$, Masayuki Itabashi ${ }^{2}$, Yoshiyuki Osamura ${ }^{2}$, Masaki Kitajima ${ }^{1}$ \\ ${ }^{1}$ Department of Gastroenterological Surgery, International University of Health and Welfare Mita Hospital, Tokyo, Japan \\ ${ }^{2}$ Department of Pathology, International University of Health and Welfare Mita Hospital, Tokyo, Japan \\ Email: "kubota@iuhw.ac.jp
}

Received 17 January 2012; revised 23 February 2012; accepted 27 February 2012

\begin{abstract}
Esophageal neuroendocrine carcinomas are rare, aggressive and have a poor prognosis. Combined therapy using chemotherapy, radiotherapy and/or surgery appear effective for them. Upper gastrointestinal endoscope of a 65-year-old male revealed a localized ulcerative lesion in the middle esophagus. Histology of biopsy specimens indicated a neuroendocrine carcinoma. A computed tomography showed an esophageal tumor with enlarged mediastinal lymph nodes. The patient was administered neoadjuvant chemotherapy consisting of 5 -fluorouracil and cisplatin, which led to partial response. Subtotal esophagectomy with three-field lymphadenectomy was performed. Pathologically, the tumor was $25 \mathrm{~mm}$ and infiltrated the proper muscle layer. The tumor cells were arranged in microtubular structures, with small and round cells containing scanty cytoplasm, and exhibited intense mitosis 51/10 HPF. They were positive for synaptophysin and chromogranin A, and Ki-67 labeling index was $70 \%-80 \%$. These findings led to the diagnosis of neuroendocrine carcinoma of small cell type. The patient was administered adjuvant chemotherapy using cisplatin and CPT-11, and he is now alive disease-free at the time of this writing.
\end{abstract}

Keywords: Esophagus; Neuroendocrine Carcinoma; Surgery; Chemotherapy

\section{INTRODUCTION}

Neuroendocrine carcinomas (NECs) of the esophagus are rare, aggressive and have a poor prognosis [1,2]. Owing to their rarity, an optimal treatment strategy has not been established, and various combinations of surgery, radiotherapy, and chemotherapy have been described [3-8]. In 2010, the World Health Organization (WHO) proposed

*Corresponding author. new diagnostic criteria for this disease that mainly depend on the rate of cellular proliferation [9].

We herein report a case of esophageal NEC and review the published literature on this rare disease.

\section{CASE REPORT}

A 65-year-old male presented with an esophageal cancer in March 2011. He had a past history of segmental gastrectomy for an early gastric cancer (well differentiated tubular adenocarcinoma) in 2007. He was a social drinker of alcohol and a former smoker. Upper gastrointestinal endoscopic examination revealed a localized ulcerative lesion (diameter, $3 \mathrm{~cm}$ ) in the middle third of the esophagus, which suspiciously invaded the proper muscle layer (Figure 1(a)). Histologic analysis of the biopsies concluded that the lesion was an NEC of the small cell type in view of round to spindle-shaped cells with scanty cytoplasm, granular nuclei, inconspicuous nucleolei and immunohistochemical evidence of neuroendocrine differentiation: many cells were reactive with synaptophysin, chromogranin A, CD56, p53, and SSTR2a, and the Ki-67 labeling index was $70 \%-80 \%$. A computed tomography scan showed an esophageal tumor with enlarged mediastinal lymph nodes along the left recurrent nerve (\#106recL) [10] (Figure 1(b)) without any abnormalities in the chest or abdominal space. The patient was therefore staged as having T2 N1 M0 esophageal cancer according to the TNM/staging classification for esophageal carcinomas [11,12]. Peripheral blood showed normal levels of all the basic biochemical parameters, including the tumor markers: CEA $1.6 \mathrm{ng} / \mathrm{mL}$; CA19-9 12 $\mathrm{U} / \mathrm{mL}$; SCC $1.0 \mathrm{ng} / \mathrm{mL}$; NSE $5.5 \mathrm{ng} / \mathrm{mL}$. No systemic hormone syndromes, such as flushing or diarrhea, were identified.

After obtaining informed consent, the patient was administered two courses of preoperative neoadjuvant chemotherapy consisting of 5-fluorouracil $\left(800 \mathrm{mg} / \mathrm{m}^{2} /\right.$ day, continuous infusion on days 1 - 5) and cisplatin $\left(80 \mathrm{mg} / \mathrm{m}^{2}\right.$ on day 1) according to the JCOG 9907 study [13]. The 


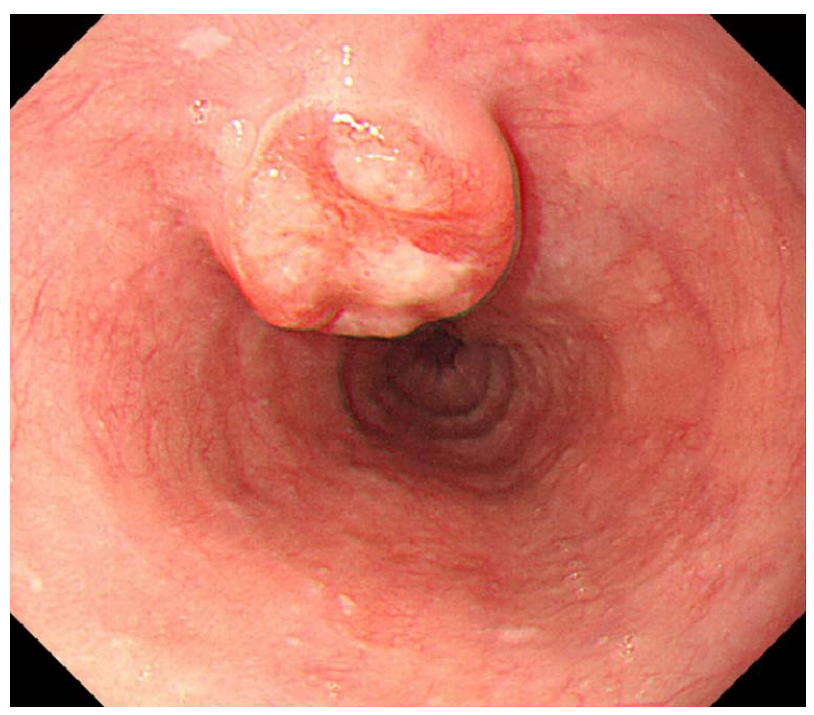

(a)

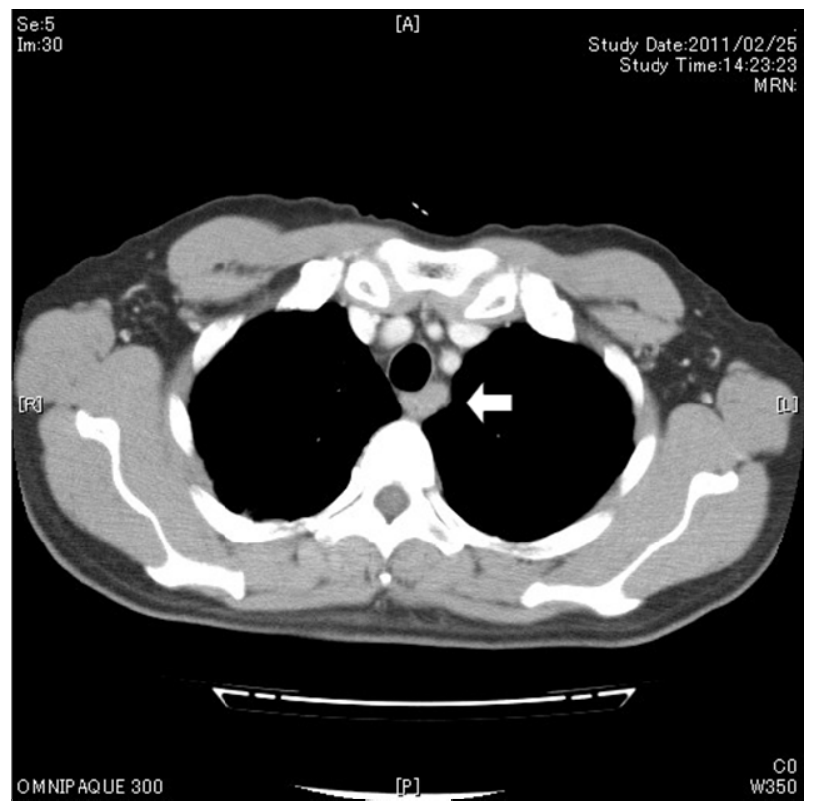

(b)

Figure 1. Upper gastrointestinal endoscopy revealed a localized ulcerative lesion (diameter, $3 \mathrm{~cm}$ ) in the middle third of the esophagus, which suspiciously invaded the proper muscle layer (a). A computed tomography scan showed an esophageal tumor with enlarged mediastinal lymph nodes (arrow) along the left recurrent nerve (b).

primary tumor at the esophagus and metastatic tumors in the lymph nodes diminished in size after the neo-adjuvant chemotherapy (Figure 2). In May 2011, subtotal esophagectomy and total gastrectomy of the remnant stomach with three-field lymphadenectomy were performed. Digestive continuity was reestablished using the right-sided colon as an esophageal substitute. Pathologically, the tumor was $25 \times 15 \mathrm{~mm}$ in size and had a negative margin. The tumor infiltrated the proper muscle

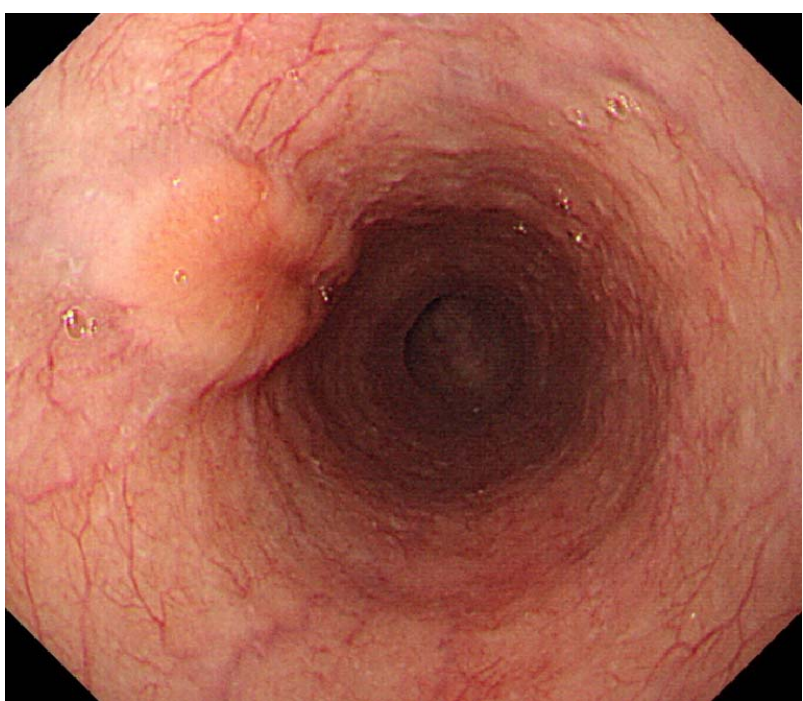

(a)

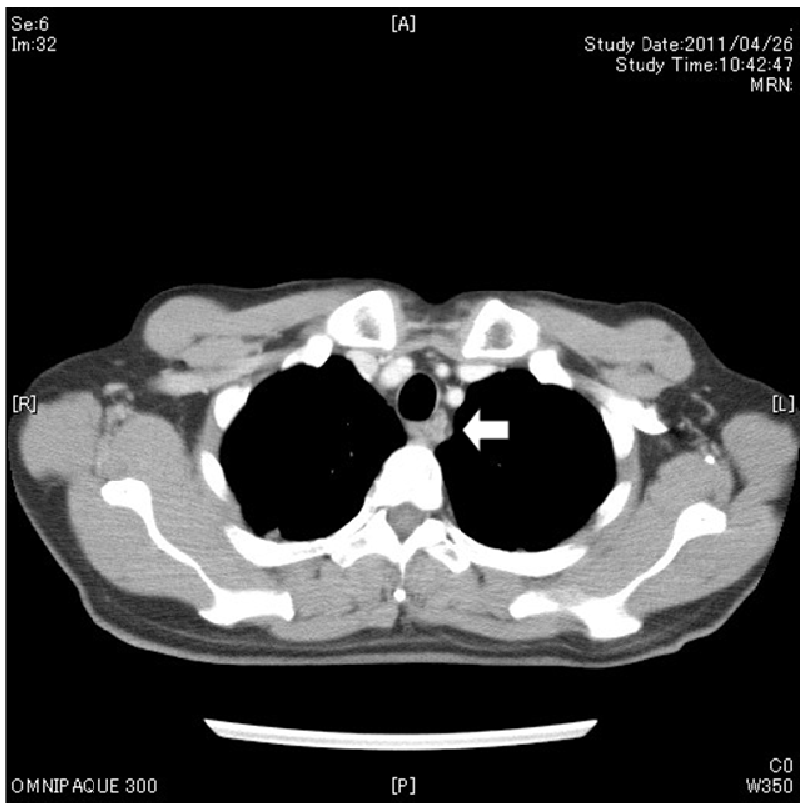

(b)

Figure 2. The primary tumor at the esophagus (a) and metastatic tumors in the lymph nodes (b, arrow) diminished in size after neoadjuvant chemotherapy.

layer. Microscopically, the tumor was uniform in shape and arranged in small microtubular structures (rosettelike arrangement) to form solid nests, with small-sized, round to cuboid tumor cells containing scanty cytoplasm (Figure 3(a)). The tumor cells exhibited hyperchromatic nuclei and intense mitosis 51/10 HPF. Lymphatic invasion was widely observed and lymph node involvement was seen in 3/53 nodes. By immunohistochemical staining, the tumor cells were positive for synaptophysin (Figure 3(b)), chromogranin A (Figure 3(c)), and CD56. The Ki-67 labeling index was 70\% - 80\% (Figure 3(d)). These 


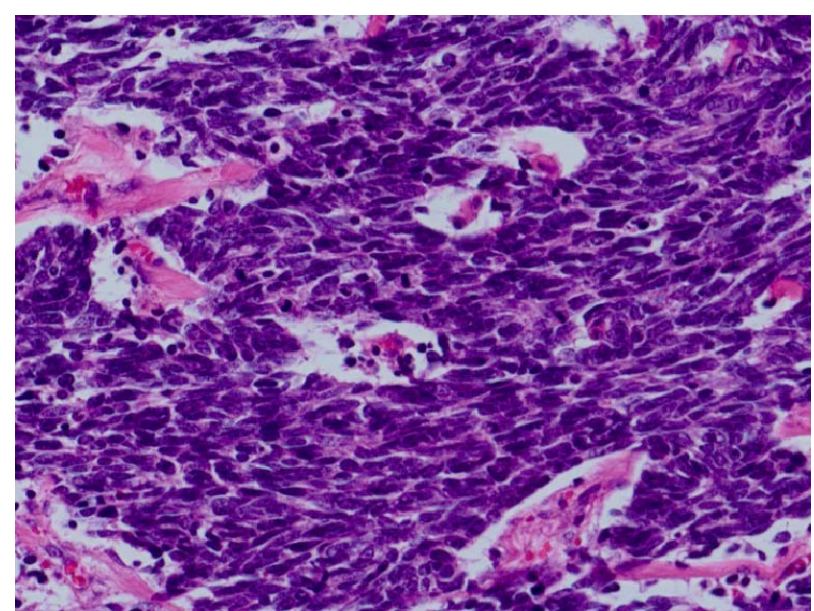

(a)

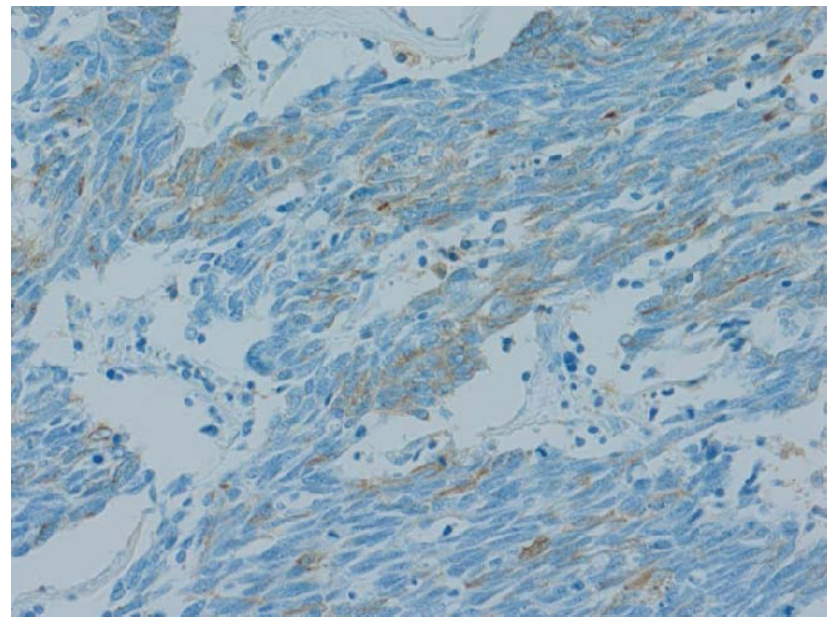

(c)

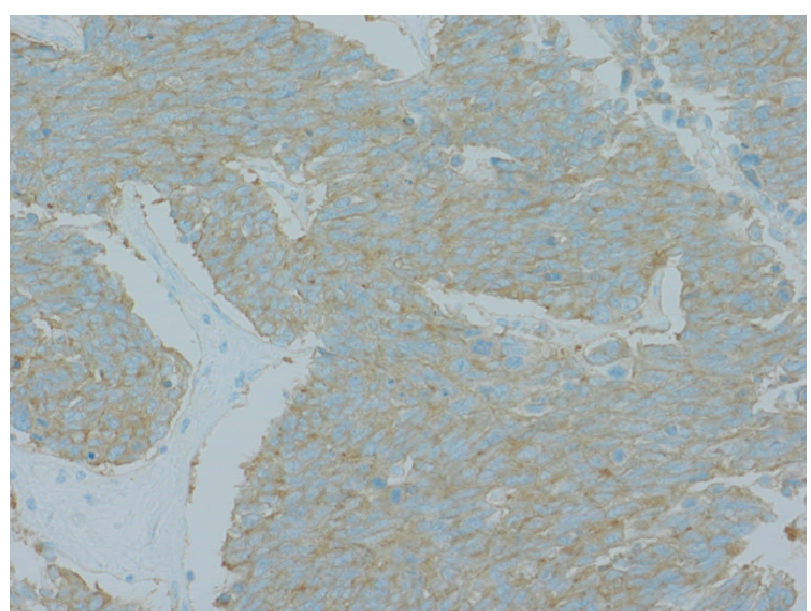

(b)

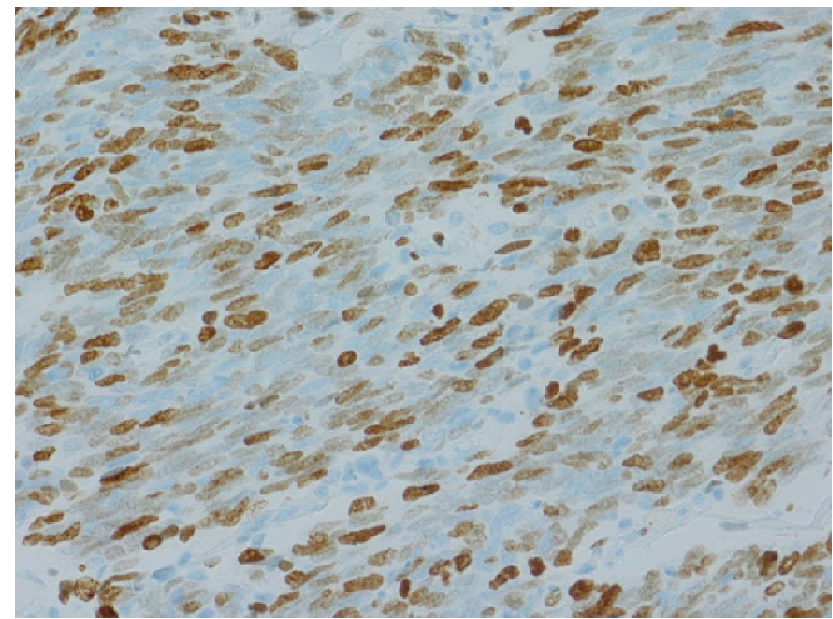

(d)

Figure 3. Microscopically, the tumor was uniform in shape and arranged in small microtubular structures (rosette-like arrangement) to form solid nests, with small, round to cuboid-shaped tumor cells containing scanty cytoplasm. Tumor cells exhibited hyperchromatic nuclei and intense mitosis $(\times 200$, a). By immunohistochemical staining, tumor cells were positive for synaptophysin (b) and chromogranin A (c). The Ki-67 labeling index was 70\% - 80\% (d).

findings led to the diagnosis of NEC of the small cell type according to the 2010 WHO criteria [9].

The postoperative course was uneventful, and the patient was discharged on the 27th day after surgery. After obtaining approval of the review board of the International University of Health and Welfare and the patient's informed consent, he was administered two courses of postoperative adjuvant chemotherapy consisting of cisplatin $\left(60 \mathrm{mg} / \mathrm{m}^{2}\right.$ on day 1$)$ and CPT-11 $\left(60 \mathrm{mg} / \mathrm{m}^{2}\right.$ on day $1,8,15)$. After that, the patient was followed-up without any maintainance therapy for 8 months without any findings indicative of recurrence or distant metastasis at the time of this writing.

\section{REVIEW OF THE LITERATURE}

Esophageal NECs are exceedingly rare. The reports include pure NEC, collision carcinoma, a composite type combined with squamous cell carcinoma or adenocarcinoma, and mixed glandular or squamous differentiation [14-16]. A pure NEC of the esophagus is extremely rare, with only about 300 cases having been reported $[17,18]$. After a literature review of 199 patients by Casas in 1997 [1], larger single-institution series have reported 9-22 patients with an esophageal NEC [3,7,19-22]. The reported frequency of these neoplasms as a proportion of all esophageal cancers ranges between $0.05 \%$ and $2.4 \%$ $[1,2]$. Regarding to small cell (endocrine) carcinoma, small cell lung cancer is a more common disease, accounting for up to $20 \%$ of all primary lung cancers and it shares many clinicopathologic features with small cell esophageal carcinoma [3]. About 5\% of the small cell carcinoma have an extrapulmonary origin, namely nasal cavities, larynx, esophagus, stomach, pancreas, and large intestine [3]. 
Esophageal NECs are aggressive neoplasms. Patients with NECs often present at an advanced stage with synchronous metastases, and the prognosis is poor [1,2]. Most esophageal NECs are large (4 - $10 \mathrm{~cm}$ in diameter), present as fungating or ulcerated masses and deeply infiltrate the esophageal wall. Almost all NECs occur in the distal half of the esophagus [1,2]. Systemic syndromes with inappropriate production of hormones, such as a syndrome of inappropriate secretion of anti-diuretic hormone and a syndrome of watery diarrhea, hypokalemia, and achlorhydria, have been reported [23,24].

Microscopically, NECs display either large cell or small cell features. Large cell NECs are more frequent, being reported in 27 out of 40 cases recently investigated, and are often associated with Barrett esophagus [1,2]. Small cell NEC of the esophagus is indistinguishable from its counterpart in the lung on the basis of histological and immunohistochemical features or clinical behavior. The cells may be small with dark nuclei having a round or oval shape and scanty cytoplasm forming solid sheets and nests. Results of immunohistochemistry for chromogranin A, synaptophysin, NCAM/CD56, B3GAT1/ Leu7 and neuron-specific enolase are usually positive, with synaptophisin being reported to be the most sensitive diagnostic marker [2,20]. Exocrine hormones, such as serotonin and gastrin, are sometimes stained, although in the absence of any hyperfunctional syndrome [18].

Recently, the concept of this disease and its diagnostic criteria has changed. In the 2010 WHO criteria, neuroendocrine neoplasms (NENs) of the esophagus are defined as neoplasms with neuroendocrine differentiation, including neuroendocrine tumors (NETs) and NECs arising in the esophagus [9]. NENs are classified into NET G1 (carcinoid), G2, and large and small cell NECs, on the basis of the level of cellular proliferation, including the mitotic and Ki-67 indices. Synonyms for NECs include poorly differentiated endocrine carcinomas, highgrade neuroendocrine carcinoma, and small cell and large cell endocrine carcinomas. In the Japanese Classification of Esophageal Cancer, endocrine cell tumors are classified into carcinoid tumor and endocrine cell carcinoma (small cell type and large cell type) [10].

In contrast to other gastrointestinal sites, there is no proposal for a TNM/staging classification for NECs of the esophagus. The relative rarity of esophageal NECs may account for this. For practical purposes, esophageal NECs may be staged according to the TNM/staging classification for esophageal carcinomas [11,12]. Despite the limited available statistics on survival, prognosis of esophageal NEC correlates reasonably with the grade and stage of the disease. Better survival was reported for patients with locoregional disease than in those with distant metastases, if treated properly [2,3].

Established treatment recommendations cannot be ex- pected for this rare entity. For the treatment of locoregional disease with curative intent, esophagectomy [5,8, $19,20,25]$ and/or radiotherapy [3,26,27] combined with adjuvant or neoadjuvant chemotherapy has been indicated in the recent literature. Cisplatin, etoposide, cyclophosphamide, and doxorubicin are backbone of most regimens for the combined chemotherapy, since chemotherapy for esophageal NEC is similar to that for small cell lung cancer, with which it shares clinicopathological similarities. A usual chemotherapy regimen for NEC of the esophagus consists of platinum doublets (including CPT-11 or etoposide). A randomized trial from Japan suggested superiority of cisplatin/irinotecan over cisplatin/etoposide for small cell lung cancer [28]. Recently, the somatostatin antagonist octreotide [29] and singleagent amrubicin chloride [30] have shown activity against NECs of the gastrointestinal tract. Results of radiotherapy [3-5] or surgery [6-8] alone generally have been disappointing and long-term survival is only rarely reported.

\section{DISCUSSION}

The concept of NEN and its diagnostic criteria have recently changed. Cancer cells from our patient had the typical histological and immunohistochemical findings of an NEC of the small cell type, according to the 2010 WHO criteria. In these criteria, NENs are classified into NETs and NECs on the basis of the mitotic and Ki-67 indices [9]. The tumor cells exhibited intense mitosis 51/10 HPF and the Ki-67 labeling index was 70\% - 80\% in our case, so he was diagnosed as NEC. Lymphatic invasion was widely observed and lymph node involvement was seen in 3/53 nodes, suggesting the high-grade malignant nature of this tumor and showing the compatibility of the diagnosis. However, our patient had a localized ulcerative lesion (diameter, $3 \mathrm{~cm}$ ) in the middle third of the esophagus and pathologically T2 N1, indicating that the NEC was found at a relatively early stage.

At first, our patient was treated with preoperative neoadjuvant chemotherapy consisting of 5-fluorouracil and cisplatin, which is usually used for esophageal squamous cell carcinomas, because there are usually various components in such tumors and a biopsy specimen might not provide complete information. The partial response to this non-specific neoadjuvant chemotherapy was gained. The surgical procedure was typical for advanced esophageal cancers. After the operation and confirmation of the histology, postoperative adjuvant chemotherapy consisting of cisplatin and CPT-11, usually used for small cell carcinoma of the lung, was administered. After that, the patient lives disease-free for 8 month at the time of this writing. A good prognosis might be expected to some extent, as this patient's tumor was a locoregional 
one and he was administered aggressive treatment with combination therapies.

In conclusion, esophageal NECs are rare and aggressive. Prognosis is poor, but survival seems to be relatively longer in patients with locoregional disease. Combined modality therapy using platinum-based combination chemotherapy and radiotherapy and/or surgery appears effective for locoregional disease, and patients with extensive disease should be treated with the same chemotherapy. We think it worth reporting a case of this rare disease, treated with multi-modality therapies and having a good prognosis expected, to increase knowledge in NECs involving gastrointestinal tract.

\section{REFERENCES}

[1] Casas, F., Ferrer, F., Farrus, B., Casals, J. and Biete, A. (1997) Primary small cell carcinoma of the esophagus: A review of the literature with emphasis on therapy and prognosis. Cancer, 80, 1366-1372.

doi:10.1002/(SICI)1097-0142(19971015)80:8<1366::AI D-CNCR2>3.3.CO;2-3

[2] Maru, D.M., Khurana, H., Rashid, A., Correa, A.M., Anandasabapathy, S., Krishnan, S., Komaki, R., Ajani, J.A., Swisher, S.G. and Hofstetter, W.L. (2008) Retrospective study of clinicopathologic features and prognosis of high-grade neuroendocrine carcinoma of the esophagus. American Journal of Surgical Pathology, 32, 1404-1411. doi:10.1097/PAS.0b013e31816bf41f

[3] Hudson, E., Powell, J., Mukherjee, S., Crosby, T.D.L., Brewster, A.E., Maughan, T.S. and Lester, J.F. (2007) Small cell esophageal carcinoma: An institutional experience and review of the literature. British Journal of Cancer, 96, 708-711. doi:10.1038/sj.bjc.6603611

[4] Huncharek, M. and Muscat, J. (1995) Small cell carcinoma of the esophagus. The massachusetts general hospital experience, 1978 to 1993. Chest, 107, 179-181. doi:10.1378/chest.107.1.179

[5] Medgysey, C.D., Wolff, R.A., Putnam Jr, J.B. and Ajani, J.A. (2000) Small cell carcinoma of the esophagus: The University of Texas M.D. Anderson Cancer Center experience and literature review. Cancer, 88, 262-267.

[6] Law, S.Y., Fok, M., Lam, K.Y., Loke, S.L., Ma, L.T. and Wong, J. (1994) Small cell carcinoma of the esophagus. Cancer, 73, 2894-2899. doi:10.1002/1097-0142(19940615)73:12<2894::AID-CN CR2820731204>3.0.CO;2-M

[7] Hosokawa, A., Shimada, Y., Matsumura, Y., Yamada, Y., Muro, K., Hamaguchi, T., Igaki, H., Tachimori, Y., Kato, H. and Shirao, K. (2005) Small cell carcinoma of the esophagus. Analysis of 14 cases and literature review. Hepato-gastroenterology, 52, 1738-1741.

[8] Craig, S.R., Carey, F.A., Walker, W.S. and Cameron, E.W. (1995) Primary small-cell cancer of the esophagus. Journal of Thoracic and Cardiovascular Surgery, 109, 284-288. doi:10.1016/S0022-5223(95)70390-X

[9] Bosman, F.T., Carneiro, F., Hruban, R.H. and Theise N.D.
(2010) WHO classification of tumors of the digestive system. 4th Edition, International Agency for Research on Cancer Publisher, Lyon.

[10] Japan Esophageal Society (2009) Japanese classification of esophageal cancer, tenth edition: Part I. Esophagus, 6, $1-25$.

[11] Sobin, L.H., Gospodarowics, M.K. and Wittekind C. (2009) TNM classification of malignant tumors. WileyBlackwell, Oxford.

[12] Edge, S.B., Byrd, D.R., Compton, C.C., Fritz, A.G., Greene, F.L. and Trotti III, A. (2009) AJCC cancer staging manual. 7th Edition, Springer, New York.

[13] Ando, N., Kato, H., Igaki, H., Shinoda, M., Ozawa, S., Shimizu, H., Nakamura, T., Yabusaki, H., Aoyama, N., Kurita, A., Ikeda, K., Kanda, T., Tsujinaka, T., Nakamura, K. and Fukuda, H. (2012) A randomized trial comparing postoperative adjuvant chemotherapy with cisplatin and 5-fluorouracil versus preoperative chemotherapy for localized advanced squamous cell carcinoma of the thoracic esophagus (JCOG9907). Annals of Surgical Oncology, 19, 68-74. doi:10.1245/s10434-011-2049-9

[14] Hamilton, K., Chiappori, A., Olson, S., Sawyers, J., Johnson, D. and Washington, K. (2000) Prevalence and prognostic significance of neuroendocrine cells in esophageal adenocarcinoma. Modern Pathology, 13, 475481. doi:10.1038/modpathol.3880081

[15] Wilson, C.I., Summerall, J., Willis, I., Lubin, J. and Inchausti, B.C. (2000) Esophageal collision tumor (large cell neuroendocrine carcinoma and papillary carcinoma) arising in a Barrett esophagus. Archives of Pathology and Laboratory Medicine, 124, 411-415.

[16] Mosnier, J.F. and Balique, J.G. (2000) Pleomorphic giant cell carcinoma of the esophagus with coexpression of cytokeratin and vimentin and neuroendocrine differentiation. Archives of Pathology and Laboratory Medicine, 124, 135-138.

[17] Takubo, K., Nakamura, K., Sawabe, M., Arai, T., Esaki, Y., Miyashita, M., Mafune, K., Tanaka, Y. and Sasajima, K. (1999) Primary undifferentiated small cell carcinoma of the esophagus. Human Pathology, 30, 216-221. doi:10.1016/S0046-8177(99)90279-4

[18] Mori, M., Matsukuma, A., Adachi, Y., Miyagahara, T., Matsuda, H., Kuwano, H., Sugimachi, K. and Enjoji, M. (1989) Small cell carcinoma of the esophagus. Cancer, 63, 564-573. doi:10.1002/1097-0142(19890201)63:3<564::AID-CNC R2820630328>3.0.CO;2-P

[19] Wu, Z., Ma, J.Y., Yang, J.J., Zhao, Y.F. and Zhang, S.F. (2004) Primary small cell carcinoma of esophagus: Report of 9 cases and review of literature. World Journal of Gastroenterology, 10, 3680-3682.

[20] Yun, J.P., Zhang, M.F., Hou, J.H., Tian, Q.H., Fu, J., Liang, X.M., Wu, Q.L. and Rong, T.H. (2007) Primary small cell carcinoma of the esophagus: Clinicopathological and immunohistochemical features of 21 cases. BMC Cancer, 7, 38. doi:10.1186/1471-2407-7-38

[21] Chin, K., Baba, S., Hosaka, H., Ishiyama, A., Mizunuma, N., Shinozaki, E., Suenaga, M., Kozuka, T., Seto, Y., Yamamoto, N. and Hatake, K. (2008) Irinotecan plus cis- 
platin for therapy of small-cell carcinoma of the esophagus: Report of 12 cases from single institution experience. Japanese Journal of Clinical Oncology, 38, 426-431. doi:10.1093/jijco/hyn041

[22] Ku, G.Y., Minsky, B.D., Rusch, V.W., Bains, M., Kelsen D.P. and Ilson, D.H. (2008) Small-cell carcinoma of the esophagus and gastroesophageal junction: Review of the memorial sloan-kettering experience. Annals of Oncology, 19, 533-537. doi:10.1093/annonc/mdm476

[23] Broicher, K. and Hienz, H.A. (1974) Carcinoid syndrome in primary esophageal tumor. Zeitschrift fur Gastroenterology, 12, 377-384.

[24] Watson, K.J., Shulkes, A., Smallwood, R.A., Douglas, M.C., Hurley, R., Kalnins, R. and Moran, L. (1985) Watery diarrhea-hypokalemia-achlorhydria syndrome and carcinoma of the esophagus. Gastroenterology, 88, 798803.

[25] Nichols, G.L. and Kelsen, D.P. (1989) Small cell carcinoma of the esophagus. The Memorial Hospital experience 1970 to 1987 . Cancer, 64, 1531-1533. doi:10.1002/1097-0142(19891001)64:7<1531::AID-CNC R2820640729>3.0.CO;2-0

[26] Yamashita, H., Nakagawa, K., Asari, T., Murakami, N., Igaki, H., Okuma, K. and Ohtomo, K. (2009) Concurrent chemoradiation alone with curative intent for limiteddisease small-cell esophageal cancer in nine Japanese patients. Diseases of the Esophagus, 22, 113-118. doi:10.1111/j.1442-2050.2008.00863.x
[27] Vos, B., Rozema, T., Miller, R.C., Hendlisz, A., Van Laethem, J.L., Khanfir, K., Weber, D.C., El Nakadi, I. and Van Houtte, P. (2011) Small cell carcinoma of the esophagus: A multicenter rare cancer network study. Diseases of the Esophagus, 24, 258-264. doi:10.1111/j.1442-2050.2010.01133.x

[28] Noda, K., Nishiwaki, Y., Kawahara, M., Negoro, S., Sugiura, T., Yokoyama, A., Fukuoka, M., Mori, K., Watanabe, K., Tamura, T., Yamamoto, S. and Saijo, N. (2002) Irinotecan plus cisplatin compared with etoposide plus cisplatin for extensive small-cell lung cancer. New England Journal of Medicine, 346, 85-91. doi:10.1056/NEJMoa003034

[29] Volante, M., Brizzi, M.P., Faggiano, A., La Rosa, S., Rapa, I., Ferrero, A., Mansueto, G., Righi, L., Garancini, S., Capella, C., De Rosa, G., Dogliotti, L., Colao, A. and Papotti, M. (2007) Somatostatin receptor type 2A immunohistochemistry in neuroendocrine tumors: A proposal of scoring system correlated with somatostatin receptor scintigraphy. Modern Pathology, 20, 1172-1182. doi:10.1038/modpathol.3800954

[30] Asayama, M., Fuse, N., Yoshino, T., Yano, T., Tahara, M., Doi, T., Fujii, S. and Ohtsu, A. (2011) Amrubicin for the treatment of neuroendocrine carcinoma of the gastrointestinal tract: A retrospective analysis of five cases. Cancer Chemotherapy and Pharmacology, 68, 13251330. doi:10.1007/s00280-011-1619-7 